\title{
Astroglial cells derived from lateral and medial midbrain sectors differ in their synthesis and secretion of sulfated glycosaminoglycans
}

G.R. Onofre ${ }^{1}$,

C.C. W erneck ${ }^{1}$,

F.A. Mendes²,

J. Garcia-Abreu²,

V. Moura Neto²,

L.A. Cavalcante 3

and L.C.F. Silva ${ }^{1}$

\author{
${ }^{1}$ Laboratório de Tecido Conjuntivo, Hospital Universitário Clementino Fraga Filho \\ and Departamento de Bioquímica Médica, ${ }^{2}$ Departamento de Anatomia, \\ and ${ }^{3}$ Programa de N eurobiologia, Instituto de Biofísica Carlos Chagas Filho, \\ Universidade Federal do Rio de Janeiro, Rio de Janeiro, RJ, Brasil
}

\section{Correspondence \\ L.C.F. Silva \\ Departamento de Bioquímica Médica CCS, UFRJ \\ Caixa Postal 68041 \\ 21941-590 Rio de Janeiro, RJ \\ Brasil \\ Fax: +55-21-270-8647 \\ E-mail: Iclaudio@ hucff.ufri.br}

Research supported by CN Pq (FINEP, PADCT and PRONEX), FAPERJ, CAPES, FUJB and CEPG/UFRJ.

Received May 15, 2000

Accepted January 3, 2001

\section{Abstract}

Astroglial cells derived from lateral and medial midbrain sectors differ in their abilities to support neuritic growth of midbrain neurons in cocultures. These different properties of the two types of cells may be related to the composition of their extracellular matrix. We have studied the synthesis and secretion of sulfated glycosaminoglycans (GAGs) by the two cell types under control conditions and B-Dxyloside-stimulated conditions, that stimulate the ability to synthesize and release GAGs. We have confirmed that both cell types synthesize and secrete heparan sulfate and chondroitin sulfate. Only slight differences were observed between the proportions of the two GAGs produced by the two types of cells after a 24-h labeling period. However, a marked difference was observed between the GAGs produced by the astroglial cells derived from lateral and medial midbrain sectors. The medial cells, which contain derivatives of the tectal and tegmental midline radial glia, synthesized and secreted $\sim 2.3$ times more chondroitin sulfate than lateral cells. The synthesis of heparan sulfate was only slightly modified by the addition of B-Dxyloside. Overall, these results indicate that astroglial cells derived from the two midbrain sectors have marked differences in their capacity to synthesize chondroitin sulfate. Under in vivo conditions or a long period of in vitro culture, they may produce extracellular matrix at concentrations which may differentially affect neuritic growth.

\section{Key words}

- Glycosaminoglycans

- Astrocytes

- Neuron-glia interactions

- Midbrain

\section{Introduction}

The central nervous system midline plays an important role in growth cone guidance by virtue of its varied cellular and molecular composition (1-3). Midline resident cells and/ or extracellular matrix (ECM) may be involved in the control of navigation of specific axon populations. One of such populations is that of the midbrain medial radial glia which differ from their lateral counterparts in their schedule of cell proliferation (4), 
cytoskeletal proteins, glycogen content, and interaction with afferent axons $(1,5-7)$. Since some distinctive features are also found in the mouse $(8,9)$, we have developed an in vitro model of astrocyte cultures of the lateral and medial sectors of the embryonic mouse midbrain (Figure 1) that differ in their ability to support neuritic growth of midbrain neurons (10). Such cultures also differ in the pattern assumed by the ECM glycoprotein laminin (11) so that neurons growing onto lateral astrocytic carpets, in which laminin displays a fibrillar configuration, extend long neurites, while neurons plated onto medial astrocytic carpets, where laminin assumes a punctate pattern, show no or only stunted processes. In addition, preliminary results suggest that astroglial cells derived from lateral and medial midbrain sectors differ in their glycosaminoglycan (GAG) composition (12). Proteoglycans form another class of macromolecules produced by astrocytes that can exert either growth-promoting or growth-inhibiting activities depending on their exact molecular composition and mode of presentation to the neurons (13-15). Many studies also suggest that the GAG parts of these molecules are responsible for effects on neuronal morphology (16-19).

We now report further characterization of the sulfated GAGs synthesized by the two types of astroglial cells. The major difference occurs in the GAGs secreted into the

Figure 1 - Photomicrograph of a cresyl violet-stained coronal section through the mouse E14 midbrain to illustrate the regions removed for cultures (dashed lines). Observe the immaturity of the midbrain, which shows neither any indication of the 3 superficial and 5 deep layers of the tectum nor the typical nuclei of the mature tegmentum. $\mathrm{M}$, Medial; L, lateral. Magnification: $63 \mathrm{X}$.

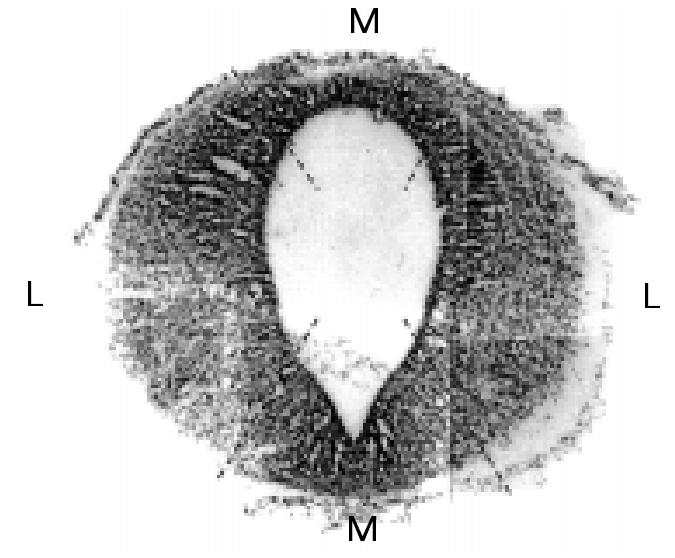

culture medium. In the presence of $\beta-D-$ xyloside, astroglial cells derived from the medial midbrain sectors secrete higher amounts of chondroitin sulfate than the cells derived from lateral midbrain. Our results show that differences in the composition and amounts of a given GAG molecule may be involved in control of axon growth through the midline.

\section{Material and Methods}

\section{Cell cultures}

Primary glial cultures from midbrains of 14-day-old Swiss mouse embryos (E14) were prepared as previously described (10). Briefly, midbrains were removed, carefully stripped off the meninges and maintained in PBS glucose. At this embryonic stage, the midbrains have the shape of a slightly curved cylinder and can first be cut as in an openbook manner. Subsequently, regions adjacent to the tectal and tegmental midline, comprising about one third of the midbrain circumference, were separated and denoted the medial region while the remaining two thirds were considered the lateral region (Figure 1). Lateral and medial regions were dissociated into single cells in a medium consisting of DMEM with F12 (Gibco, Gaithersburg, MD, USA) enriched with glucose, glutamine and sodium bicarbonate as indicated above. Dissociated cells were plated with the same medium as for dissociation, supplemented with $10 \%$ FCS. Cultures were kept for 3 weeks, with changes of the DMEMF12-10\% FCS medium every 3-4 days.

Repeated analyses of the confluent cultures with antibodies binding to astrocytes (anti-GFAP), neurons (anti-beta 3 tubulin) or oligodendrocytes (anti-CNPase) as well as tests with an isolectin (BS1B4 of Griffonia simplicifolia) that recognizes microglial cells have shown that virtually all cells in either type of culture are astrocytes (see Figure 2 in Refs. 10 and 20). 
Labeling of sulfated GAGs from lateral and medial glial cell cultures

Lateral and medial glial cell cultures that reached confluence were treated with $30 \mu \mathrm{Ci}$ of $\mathrm{H}_{2}{ }^{35} \mathrm{SO}_{4}$ in DMEM-F12-10\% FCS medium. Cultures were incubated in this medium at $37^{\circ} \mathrm{C}$ in a humidified atmosphere containing $5 \% \mathrm{CO}_{2}$ for $24 \mathrm{~h}$.

\section{Isolation of sulfated GAGs from lateral or medial glial cell cultures}

Sulfated GAGs were isolated from the pericellular, intracellular and extracellular compartments of astrocyte cultures from lateral and medial sectors of embryonic mouse midbrain as previously described for other types of cells $(21,22)$, as follows.

Essentially, at the completion of the labeling period, conditioned media containing either the lateral or medial soluble extracellular GAGs were removed and centrifuged (1000 rpm for $5 \mathrm{~min}$ ) to remove any cell debris and stored at $-20^{\circ} \mathrm{C}$ until required. The cells were rinsed with PBS, $\mathrm{pH}$ 7.4, being detached by incubation with $2 \mathrm{ml}$ of $0.25 \%(\mathrm{w} / \mathrm{v})$ trypsin and $0.05 \%$ EDTA in PBS for $10 \mathrm{~min}$ at $37^{\circ} \mathrm{C}$. Centrifugation of the trypsin-treated cells $(2,000 \mathrm{~g}$ for $10 \mathrm{~min}$ at room temperature) separated the supernatant and cell pellet, which contained the pericellular and intracellular GAGs, respectively. These fractions from lateral and medial glial cultures were incubated again for $12 \mathrm{~h}$ at $37^{\circ} \mathrm{C}$ with $2 \mathrm{ml} \mathrm{PBS}, \mathrm{pH} 7.4$, containing $0.05 \%$ trypsin and $0.05 \%$ EDTA, and then dialyzed against distilled water. The samples were further incubated with $10 \mathrm{mg}$ papain (Sigma Chemical Co., St. Louis, MO, USA) in the presence of $5 \mathrm{mM}$ EDTA and 5 $\mathrm{mM}$ cysteine at $60^{\circ} \mathrm{C}$ for $24 \mathrm{~h}$. The ${ }^{35} \mathrm{~S}-$ labeled GAGs were purified on a DEAEcellulose column, as described below.

For isolation of extracellular GAGs, the previously collected lateral or medial incubation medium was incubated with $10 \mathrm{mg}$ papain in the presence of $5 \mathrm{mM}$ EDTA and 5 $\mathrm{mM}$ cysteine at $60^{\circ} \mathrm{C}$ for $24 \mathrm{~h}$, dialyzed against distilled water, and applied to a DEAE-cellulose column $(3.5 \times 2.5 \mathrm{~cm})$ equilibrated with $50 \mathrm{mM}$ sodium acetate buffer, pH 5.0. The column was washed with $100 \mathrm{ml}$ of the same buffer and then eluted stepwise with $25 \mathrm{ml}$ of $0.1 \mathrm{M} \mathrm{NaCl}$ in the same acetate buffer. The ${ }^{35} \mathrm{~S}-\mathrm{GAGs}$ were eluted from the column with $1.0 \mathrm{M} \mathrm{NaCl}$ and exhaustively dialyzed against distilled water. The dialyzed GAG samples from lateral or medial media were lyophilized and dissolved in 0.2 $\mathrm{ml}$ distilled water.

\section{Agarose gel electrophoresis}

Agarose gel electrophoresis was carried out as previously described (22). Approximately $1,000 \mathrm{cpm}$ of ${ }^{35} \mathrm{~S}-\mathrm{GAGs}$ from cellassociated fractions or secreted into the culture medium of lateral and medial glial cell cultures before and after chondroitin lyase digestion or deaminative cleavage with nitrous acid, as well as a mixture of standard chondroitin 4-sulfate, dermatan sulfate and heparan sulfate ( $10 \mu \mathrm{g}$ of each) were applied to $0.5 \%$ agarose gels in $0.05 \mathrm{M} \mathrm{1,3-di-}$ aminopropane:acetate, $\mathrm{pH}$ 9.0. After electrophoresis, GAGs were fixed in the gel with $0.1 \% N$-cetyl- $N, N, N$-trimethylammonium bromide in water, and stained with $0.1 \%$ toluidine blue in acetic acid:ethanol:water (0.1:5:5, v/v/v). The ${ }^{35} \mathrm{~S}$-labeled GAGs were visualized by autoradiography of the stained gels. The radioactive bands having identical electrophoretic migration with standard GAGs were carefully scraped into $10 \mathrm{ml}$ of a $0.5 \% \mathrm{PPO} /$ toluene solution and counted in a liquid scintillation counter.

\section{Enzymatic and nitrous acid depolymerization of the GAGs}

Digestion with chondroitin lyases. Digestion with chondroitin $\mathrm{AC}$ or $\mathrm{ABC}$ lyases was carried out by the method of Saito et al. 
(23). Approximately $10,000 \mathrm{cpm}$ of ${ }^{35} \mathrm{~S}-\mathrm{la}-$ beled GAGs were incubated with 0.3 units of chondroitin AC lyase or chondroitin $\mathrm{ABC}$ lyase for $8 \mathrm{~h}$ at $37^{\circ} \mathrm{C}$ in $100 \mu 1$ of $50 \mathrm{mM}$ Tris$\mathrm{HCl}, \mathrm{pH} 8.0$, containing $5 \mathrm{mM}$ EDTA and 15 $\mathrm{mM}$ sodium acetate.

Deamination with nitrous acid. Deamination with nitrous acid at $\mathrm{pH} 1.5$ was performed as described by Shively and Conrad (24). Briefly, approximately $10,000 \mathrm{cpm}$ of ${ }^{35}$ S-labeled GAGs were incubated with 200 $\mu \mathrm{l}$ of freshly generated $\mathrm{HNO}_{2}$ at room temperature for $10 \mathrm{~min}$. The reaction mixtures were then neutralized with $1.0 \mathrm{M} \mathrm{Na}_{2} \mathrm{CO}_{3}$.

\section{Analysis of the ${ }^{35}$ S-disaccharides formed by enzymatic depolymerization of extracellular ${ }^{35} \mathrm{~S}-\mathrm{chondroitin}$ sulfate from lateral and medial glial cultures}

Purified extracellular radiolabeled sulfated GAGs were submitted to exhaustive digestion with chondroitin $\mathrm{AC}$ or $\mathrm{ABC}$ lyases
Table 1 - Incorporation of ${ }^{35} \mathrm{~S}$-sulfate into specific glycosaminoglycans (GAGs) from cell-associated and secreted fractions of lateral and medial glial cells incubated in the absence or presence of ß-D-xyloside.

Cultures of glial cells were incubated for $24 \mathrm{~h}$ with $30 \mu \mathrm{Ci} \mathrm{H}{ }_{2}{ }^{35} \mathrm{SO}_{4}$ in the presence or absence of $0.5 \mathrm{mM}$ ß-D-xyloside. GAGs from cells and medium were isolated and analyzed by agarose gel electrophoresis. The ${ }^{35} \mathrm{~S}-\mathrm{GAGs}$ were visualized by autoradiography of the stained gels. The radioactive bands having electrophoretic migration identical to that of standard GAGs were carefully scraped into $10 \mathrm{ml}$ of $0.5 \%$ PPO/ toluene solution and counted in a liquid scintillation counter. CS, Chondroitin sulfate; HS, heparan sulfate. Values in parentheses are the ratio of each GAG within each compartment after/before ß-D-xyloside treatment.

\begin{tabular}{|c|c|c|c|c|c|}
\hline Glial type & Additions & GAG & $\begin{array}{l}\text { Total } \\
\text { (cpm) }\end{array}$ & $\begin{array}{l}\text { Cell-associated } \\
\text { (cpm) }\end{array}$ & $\begin{array}{c}\text { Culture } \\
\text { medium (cpm) }\end{array}$ \\
\hline \multicolumn{6}{|c|}{ a) Without B-D-xyloside } \\
\hline \multirow[t]{2}{*}{ Lateral } & None & CS & 4,806 & 1,382 & 3,424 \\
\hline & & HS & 6,012 & 4,927 & 1,085 \\
\hline \multirow[t]{2}{*}{ Medial } & None & CS & 3,711 & 1,323 & 2,388 \\
\hline & & HS & 6,867 & 5,452 & 1,415 \\
\hline \multicolumn{6}{|c|}{ b) In the presence of ß-D-xyloside } \\
\hline \multirow[t]{2}{*}{ Lateral } & Xyloside & $\mathrm{CS}$ & 18,488 & $7,668(5.55)$ & $10,820(3.16)$ \\
\hline & & HS & 8,771 & $5,856(1.21)$ & $2,915(2.69)$ \\
\hline \multirow[t]{2}{*}{ Medial } & Xyloside & CS & 29,766 & $5,098(3.85)$ & $24,668(10.33)$ \\
\hline & & HS & 6,216 & $4,494(0.82)$ & $1,722(1.22)$ \\
\hline
\end{tabular}

(see above). After incubation, each mixture was applied to Whatman No. 1 chromatography paper and developed in isobutyric acid: $1 \mathrm{M} \mathrm{NH}_{4} \mathrm{OH}(5: 3, \mathrm{v} / \mathrm{v})$ for $24 \mathrm{~h}$. The ${ }^{35} \mathrm{~S}-$ labeled degradation products were detected by autoradiography of the chromatograms. The radioactive bands with chromatographic migration identical to that of standard disaccharides $\left(\alpha-\Delta\right.$ GlcUA- $\rightarrow 3$-GalNAc $\left(4 \mathrm{SO}_{4}\right)$ and $\alpha-\Delta$ GlcUA- $\left.1 \rightarrow 3-\operatorname{GalNAc}\left(6 \mathrm{SO}_{4}\right)\right)$ were cut out and counted in $10 \mathrm{ml}$ of $\mathrm{PPO} /$ toluene.

\section{Effects of methyl ß-D-xyloside on GAG synthesis}

Lateral and medial glial cells were labeled for $24 \mathrm{~h}$ with $\mathrm{H}_{2}{ }^{35} \mathrm{SO}_{4}$ as described above, with or without $0.5 \mathrm{mM}$ methyl $\beta-\mathrm{D}$ xyloside. At the end of the labeling period the secreted and cell-associated GAGs were analyzed by agarose gel electrophoresis as described above (see also Ref. 21).

\section{Results and Discussion}

We analyzed the amounts of ${ }^{35} \mathrm{~S}$-labeled GAGs found in cell-associated fractions and those secreted into the culture medium by astrocyte cells derived from lateral and medial midbrain sectors after a 24-h labeling period with ${ }^{35} \mathrm{~S}^{3} \mathrm{Na}_{2} \mathrm{SO}_{4}$. Table 1a shows that both glial cultures expressed heparan sulfate and chondroitin sulfate in the two cellular compartments, but heparan sulfate was more abundant in the cell-associated fractions, while chondroitin sulfate predominated in the culture medium. However, it is possible to argue that the astroglial cells under in vitro culture conditions are not stimulated to synthesize GAG chains at the same intensity as observed in vivo. Another alternative is that the results reported in Table 1 for cells obtained from lateral and medial midbrain sectors may not reflect the capacity of the two cell types to synthesize GAGs, but rather the availability of the protein core required to initiate glycan assembly. In order 
to investigate these possibilities we stimulated GAG synthesis by the addition of B-Dxyloside to the culture medium. This agent greatly stimulates chondroitin sulfate chain synthesis and secretion $(21,25,26)$. This procedure allowed us to evaluate more precisely the capacity of the two cell types to secrete chondroitin sulfate chains.

The addition of B-D-xyloside to cell cultures has been used as an enhancement technique to approach a different problem. Brachymorphic mice present a metabolic defect, which results in a deficient sulfation of the chondroitin sulfate chains. The defect is easily detected in cartilage due to its rapidly growing rate but not in skin fibroblast cultures. However, the addition of $B-D-$ xyloside to the cell cultures and the consequent increase in the synthesis of chondroitin sulfate clearly demonstrated a deficiency in the sulfation of this GAG (26).

The addition of B-D-xyloside led to a greater secretion of GAGs by astrocytes from medial midbrain sectors compared to lateral midbrain sectors (Table 2).

We also investigated the effect of B-Dxyloside on the different types of GAG chains (Table 1b). The increased synthesis of GAGs in the presence of $B-D$-xyloside was always more intense for chondroitin sulfate than for heparan sulfate chains within each compartment of the two types of cell cultures (Table 1b). However, more significantly, the increased synthesis of chondroitin sulfate induced by $\beta-D$-xyloside was much more intense for cells derived from the medial than from the lateral midbrain sectors. The amounts of ${ }^{35} \mathrm{~S}$-sulfate incorporated into chondroitin sulfate chains secreted into the culture medium by lateral and medial midbrain glial cells increased 3.16- and 10.33fold, respectively. The synthesis of heparan sulfate was not dramatically modified by the addition of $\beta$-D-xyloside, and in the case of the cell-surface compartment of the astrocytes from medial midbrain it was not increased at all, but slightly decreased
(Table 1b).

The addition of $B$-D-xyloside to the astrocyte cultures emphasizes the difference between the synthesis of GAGs by cells derived from the lateral and medial midbrain sectors. Cells from the medial midbrain have a markedly increased capacity to synthesize and secrete chondroitin sulfate when compared with cells obtained from the lateral midbrain.

We already reported preliminary results indicating that astroglial cells derived from lateral and medial midbrain sectors differ in their GAG composition (12). However, this initial study suggested exclusively differences in the proportion of heparan sulfate and chondroitin sulfate in the cell-associated and extracellular compartments. In the present work we expanded this study. We now report an additional difference between midbrain and lateral astrocytes, not only in GAG composition, but in the ability of midbrain astrocytes to synthesize and secrete larger amounts of one of the GAGs, chondroitin sulfate, compared to lateral glia.

Since astroglial cells derived from the lateral and medial midbrain sectors differ in their capacity to synthesize and secrete chondroitin sulfate into the culture medium, we decided to determine whether these two cell types also differ in the structure of their GAG chains. ${ }^{35} \mathrm{~S}-\mathrm{GAG}$ from culture medi-

Table 2 - Effect of ß-D-xyloside on the cell-associated and secreted ${ }^{35} \mathrm{~S}$-labeled glycosaminoglycans (GAGs) from lateral and medial glial cells.

Cultures of glial cells were incubated for $24 \mathrm{~h}$ with $30 \mu \mathrm{Ci} \mathrm{H}{ }^{35} \mathrm{SO}_{4}$ in the presence or absence of $0.5 \mathrm{mM}$ ß-D-xyloside. GAGs from cells and medium were analyzed by agarose gel electrophoresis as described in Table 1 . Values in parentheses are the ratio of total GAGs within each compartment after/before ß-D-xyloside treatment.

\begin{tabular}{lccccc}
\hline Glial type & Additions & $\begin{array}{c}\text { Total } \\
(\mathrm{cpm})\end{array}$ & $\begin{array}{c}\text { Cell-associated } \\
(\mathrm{cpm})\end{array}$ & $\begin{array}{c}\text { Secreted } \\
(\mathrm{cpm})\end{array}$ & $\begin{array}{c}\text { Ratio secreted/ } \\
\text { cell-associated }\end{array}$ \\
\hline Lateral & None & 10,818 & 6,309 & 4,509 & 0.71 \\
& Xyloside & 27,559 & $13,524(2.14)$ & $13,735(3.05)$ & 1.02 \\
Medial & None & 10,578 & 6,775 & 3,803 & 0.56 \\
& Xyloside & 35,982 & $9,592(1.42)$ & $26,390(6.96)$ & 2.75
\end{tabular}


um of lateral and medial cells were submitted to exhaustive digestion with chondroitin $\mathrm{AC}$ and $\mathrm{ABC}$ lyases. The disaccharides formed were analyzed by paper chromatography (Table 3). GlcUA-GalNAc $\left(4 \mathrm{SO}_{4}\right)$ accounts for about $80 \%$ of total disaccharide units found in the chondroitin sulfate synthesized by the two astroglial cells. No marked differences were observed in the disaccharide composition of the GAGs obtained from lateral and medial cells, except for a slight decrease in GlcUA-GalNAc $\left(4 \mathrm{SO}_{4}\right)$ and a concomitant increase in IdUAGalNAc $\left(4 \mathrm{SO}_{4}\right)$ in the chondroitin sulfate derived from medial cells. Thus, a minor amount of iduronic acid-containing disaccharide units (typical of dermatan sulfate) was also detected on the structure of extracellular chondroitin sulfate. Additional studies are necessary in order to clarify whether this iduronic acid unit is a constituent of the astroglial chondroitin sulfate, or if it constitutes a different polymer. Minor amounts of dermatan sulfate have been reported in astrocyte cultures from rat postnatal cerebral hemispheres (27).

Astrocytes, the major glial population in the nervous system, are characterized by their expression of glial fibrillary acidic protein. Recent studies have demonstrated that glial cells are also among the most function-

Table 3 - Proportions of the disaccharide units from the extracellular chondroitin sulfate of lateral and medial glial cells.

The values in parentheses represent the percentages of the disaccharide units of the chondroitin sulfate chains. The proportions of the various disaccharide units were determined based on the combined digestion of the GAG with chondroitin $A C$ and $A B C$ lyases (see Ref. 22).

\begin{tabular}{lccc}
\hline Glial type & \multicolumn{3}{c}{ Disaccharide units (cpm) } \\
\cline { 2 - 4 } & $\begin{array}{c}\text { GlcUA-GalNAc4S } \\
\text { (from chondroitin } \\
\text { 4-sulfate) }\end{array}$ & $\begin{array}{c}\text { GlcUA-GalNAc6S } \\
\text { (from chondroitin } \\
\text { 6-sulfate) }\end{array}$ & $\begin{array}{c}\text { IdUA-GalNAc4S } \\
\text { (from dermatan } \\
\text { sulfate) }\end{array}$ \\
\hline Lateral & $12,212(85 \%)$ & $569(5 \%)$ & $1,522(10 \%)$ \\
Medial & $6,921(72 \%)$ & $766(8 \%)$ & $1,922(20 \%)$
\end{tabular}

ally diverse groups of cells in the nervous system both during early development and in adults. These cells are known to synthesize and release multiple molecules, including peptide growth factors (NGF, FGF) and ECM proteins such as laminin and fibronectin. Proteoglycans are also among these astrocyte-derived neuroactive molecules, which have been suggested to play important roles in the regulation of cellular differentiation, neurite outgrowth and synaptogenesis (reviewed by Dow and Wang (15)). However, the functional significance of proteoglycans produced by astrocytes has yet to be fully elucidated. A large number of proteoglycans have been implicated in the regulation of neurite outgrowth, providing signals that either promote or inhibit neurite outgrowth. Traditionally, the expression of heparan sulfate proteoglycans has been associated with neuritogenesis, while chondroitin sulfate proteoglycans appeared to provide inhibitory cues to growing neurites. However, it is not possible to establish a clear classification (28). In fact, there are clear examples of chondroitin sulfate proteoglycans which provide promoting cues to the growth cones, while some heparan sulfate proteoglycans can interfere with growth cone movements and even induce growth cone collapse and retraction (28, see also references within). Heparan sulfate proteoglycans anchored to the cell surface may function as receptors for ECM components and bind growth factors such as FGF or NGF (15). Ard and Bunge (29) have observed colocalization of heparan sulfate proteoglycans and laminin on the astrocyte surface in vitro and have noted that growing neurites contacted areas where these complexes were deposited. However, exogenous heparan sulfate can inhibit neurite growth in neurons growing on an L1 substrate (30). Furthermore, Garcia-Abreu et al. (19) have shown that heparan sulfate contributes to the nonpermissive role of the midline glia in the growth of midbrain neurites.

Chondroitin sulfate has been considered 
to be inhibitory for neurite growth on the basis of the effects of non-neural GAGs as substrate (16) and of a correlation between its marked accumulation in the tectal midline and a barrier function for retinal axons (31). However, some axon systems grow on a chondroitin proteoglycan path in vivo (32). Furthermore, the DSD-1 proteoglycan and phosphacan, both of which are chondroitin sulfate proteoglycans, promote neurite outgrowth from midbrain and hippocampal neurons and this effect is mediated by the GAG structure $(33,34)$. Chung et al. (35) have recently reported that the axon routing at the optic chiasm changes after enzymatic removal of chondroitin sulfate in mouse embryos and that navigation of normally projecting ipsilateral axons is perturbed, suggesting that chondroitin sulfate is involved in patterning the early phase of axonal growth across the midline and at a later stage controlling the axon divergence at the chiasm.

With this in vitro model using astrocyte cultures of the lateral and medial sectors from embryonic mouse midbrain, we have previously demonstrated that glial cells derived from different sectors of the midbrain differ in their ability to support neuritic growth of midbrain neurons and in the pattern assumed by the ECM glycoprotein laminin $(10,11)$. Neurons growing onto lateral astrocytic carpets, in which laminin displays a fibrillar configuration, extend long neurites, while neurons plated onto medial astrocytic carpets, where laminin assumes a punctate pattern, show only stunted or no processes. We also suggest that these differential glial properties might be, at least in part, ascribed to the differential ability to synthesize and/or to compartmentalize and/or to secrete GAGs (12). Recently we observed that the GAG chain rather than the intact proteoglycan molecule might be responsible for the differential effects of the two glial types on neurite growth (19).

Available information about our culture system suggests that chondroitin sulfate might not be responsible for the nonpermissive properties of medial glia since treatment of this glial type with chondroitin lyase had little functional effect (19). In fact, the enzymatic treatment caused a significant reduction in the growth-supporting ability of lateral glia. Clearly, functional experiments with xyloside-treated cultures may help clarify the role of glycosaminoglycans in the growthmodulating properties of lateral and medial midbrain glia.

\section{Acknowledgments}

Dr. Paulo A.S. Mourão is gratefully acknowledged for a critical reading of this manuscript. We also thank Sergio L. Carvalho for assistance with the photomicrography.

\section{References}

1. Barradas PC, Cavalcante LA, MendezOtero R \& Vieira AM (1989). Astroglial differentiation in the opossum superior colliculus. Glia, 2: 103-111.

2. SilverJ (1993): Glia-neuron interactions at the midline of the developing mammalian brain and spinal cord. Perspectives on Developmental Neurobiology, 1: 227-236.

3. Wang LC, Dani J , Godement P, Marcus R \& Mason CA (1995). Crossed and uncrossed retinal axons respond differently to cells of the optic chiasm midline in vitro. Neuron, 15: 1349-1364.
4. Raedler E, Raedler A \& Feldhaus S (1981). Prenatal differentiation of superior colliculus in the rat. Bibliotheca Anatomica, 19: 174-191.

5. J haveri S (1993). Midline glia of the tectum: a barrier for developing retinal axons. Perspectives on Developmental Neurobiology, 4: 237-243.

6. Pulido-Caballero $\mathrm{J}, \mathrm{J}$ imenez-Sampedro $\mathrm{F}$, Echevarria-Aza D \& Martinez-Millan L (1994). Postnatal development of vimentin-positive cells in the rabbit superior colliculus. J ournal of Comparative Neurology,
343: 102-112.

7. Wu DY, Schneider GE, Silver J , Poston M \& J haveri S (1998). A role for tectal midline glia in the unilateral containment of retinocollicular axons. J ournal of Neuroscience, 18: 8344-8355.

8. Edwards MA, Yamamoto $M \&$ Caviness J r VS (1990). Organization of radial glia and related cells in the developing murine CNS. An analysis based upon a new monoclonal antibody marker. Neuroscience, 36: 121-144.

9. Hamre KM, Chepenik KP \& Goldowitz D 
(1995). The annexins: specific markers of midline structures and sensory neurons in the developing murine central nervous system. J ournal of Comparative Neurology, 352: 421-435.

10. Garcia-Abreu J , Moura Neto V, Carvalho SL \& Cavalcante LA (1995). Regionally specific properties of midbrain glia: I. Interactions with midbrain neurons. J ournal of Neuroscience Research, 40: 471-477.

11. Garcia-Abreu J, Cavalcante LA \& Moura Neto V (1995). Differential pattems of laminin expression in lateral and medial midbrain glia. NeuroReport, 6: 761-764.

12. Garcia-Abreu J, Silva LCF, Tovar FF, Onofre GR, Cavalcante LA \& Moura Neto $\checkmark$ (1996). Compartmental distribution of sulfated glycosaminoglycans in lateral and medial midbrain astroglia cultures. Glia, 17: 339-344.

13. Stipp CS, Litwack ED \& Lander AD (1994). Cerebroglycan: an integral membrane heparan sulfate proteoglycan that is unique to the developing nervous system and expressed specifically during neuronal differentiation. J ournal of Cell Biology, 124: 149-160.

14. Halfter W, Schurer B, Yip J, Yip L, Tsen G, Lee J A \& Cole GJ (1997). Distribution and substrate properties of agrin, a heparan sulfate proteoglycan of developing axonal pathways. J ournal of Comparative Neurology, 383: 1-17.

15. Dow KE \& Wang W (1998). Cell biology of astrocyte proteoglycans. Cellular and Molecular Life Sciences, 54: 567-581.

16. Snow DM, Lemmon V, Carrino DA, Caplan Al \& Silver J (1990). Sulfated proteoglycans in astroglial barriers inhibit neurite outgrowth in vitro. Experimental Neurology, 109: 111-130.

17. Lafont $F$, Rouget $M$, Triller $A$, Prochiantz $A \&$ Rousselet A (1992). In vitro control of neuronal polarity by glycosaminoglycans. Development, 114: 17-29.

18. Bovolenta $P$, Fernaud-Espinosa I, Mendez-Otero R \& Nieto-Sampedro M (1997). Neurite outgrowth inhibitor of gliotic brain tissue. Mode of action and cellular localization, studied with specific monoclonal antibodies. European J ournal of Neuroscience, 9: 977-989.

19. Garcia-Abreu J, Mendes FA, Onofre GR, Freitas MS, Silva LCF, Moura Neto V \& Cavalcante LA (2000). Contribution of heparan sulfate to the non-permissive role of the midline glia to the growth of midbrain neurites. Glia, 29: 260-272.

20. Garcia-Abreu J, Cavalcante LA, Silva LCF \& Moura Neto V (1996). The extracellular matrix of the midline and non-midline midbrain glia: correlations with neurite growth-supporting abilities. Brazilian J ournal of Medical and Biological Research, 29: 1179-1187.

21. Silva LCF, Borojevic R \& Mourão PAS (1992). Membrane-associated and secreted proteoglycans from a continuous cell line derived from fibrotic schistosomal granulomas. Biochimica et Biophysica Acta, 1138: 133-142.

22. Werneck CC, Oliveira-dos-Santos AJ, Silva LCF, Villa-Verde DMS, Savino W \& Mourão PAS (1999). Thymic epithelial cells synthesized a heparan sulfate with highly sulfated region. J ournal of Cellular Physiology, 178: 51-62.

23. Saito N, Yamagata T \& Suzuki S (1968). Enzymatic methods for the determination of small quantities of isomeric chondroitin sulfates. J ournal of Biological Chemistry, 243: 1536-1544.

24. Shively JE \& Conrad HE (1976). Formation of anhydrosugars in the chemical depolymerization of heparin. Biochemistry, 15: 3932-3942.

25. Lugemwa FN \& Esko J D (1991). Estradiol beta-D-xyloside, an efficient primer for heparan sulfate biosynthesis. J ournal of Biological Chemistry, 266: 6674-6677.

26. Pennypacker J P, Kimata K \& Brown KS (1981). Brachymorphic mice (bm/bm): a generalized biochemical defect expressed primarily in cartilage. Developmental Biology, 81: 280-287.

27. J ohnson-Green PC, Dow KE \& Riopelle
RJ (1991). Characterization of glycosaminoglycans produced by primary astrocytes in vitro. Glia, 4: 314-321.

28. Bovolenta P \& Fernaud-Espinosa I (2000). Nervous system proteoglycans as modulators of neurite outgrowth. Progress in Neurobiology, 61: 113-132.

29. Ard MD \& Bunge RP (1988). Heparan sulphate proteoglycans and laminin immunoreactivity on cultured astrocytes: relationship to differentiation and neurite growth. J ournal of Neuroscience, 8: 28442858.

30. Dou CJ \& Levine J M (1995). Differential effects of glycosaminoglycans on neurite growth on laminin and L1 substrates. J ournal of Neuroscience, 15: 8053-8066.

31. Hoffman-Kim D, Lander $A \&$ J haveri $S$ (1998). Patterns of chondroitin sulfate immunoreactivity in the developing tectum reflect regional differences in glycosaminoglycan biosynthesis. J ournal of Neuroscience, 18: 5881-5890.

32. Sheppard AM, Hamilton SK \& Pearlman AL (1991). Changes in the distribution of extracellular matrix components accompany early morphogenetic events of mammalian cortical development. J ournal of Neuroscience, 11: 3928-3942.

33. Faissner $A$, Clement $A$, Lochter $A$, Streit A, Mandl C \& Schachner M (1994). Isolation of a neural chondroitin sulfate proteoglycan with neurite outgrowth promoting properties. J ournal of Cell Biology, 126: 783-799.

34. Garwood J , Schnädelbach O, Clement A Schütte K, Bach A \& Faissner A (1999). DSD-1 proteoglycan is the mouse homologue of phosphacan and displays opposing effects on neurite outgrowth dependent on neuronal lineage. J ournal of Neuroscience, 19: 3888-3899.

35. Chung KY, Taylor J S, Shum DK \& Chan SO (2000). Axon routing at the chiasm after enzymatic removal of chondroitin sulfate in mouse embryos. Development, 127: 2673-2683. 